\title{
Design and characterization of quantum dot photonic crystal lasers
}

Tomoyuki Yoshie, Oleg B. Schchekin, Hao Chen, Dennis G. Deppe, Axel Scherer

Tomoyuki Yoshie, Oleg B. Schchekin, Hao Chen, Dennis G. Deppe, Axel Scherer, "Design and characterization of quantum dot photonic crystal lasers," Proc. SPIE 5000, Photonic Crystal Materials and Devices, (9 July 2003); doi: 10.1117/12.479515

SPIE Event: Integrated Optoelectronics Devices, 2003, San Jose, CA, United States 


\title{
Design and characterization of quantum dot photonic crystal lasers
}

\author{
Tomoyuki Yoshie $^{a}$, Oleg B. Schchekin ${ }^{b}$, Hao $_{\text {Chen }}{ }^{b}$, Dennis G. Deppe ${ }^{b}$, and Axel Scherer ${ }^{a}$ \\ ${ }^{a}$ Department of Electrical Engineering, Caltech, Pasadena CA 91125-9300 U.S.A. \\ ${ }^{b}$ Microelectronics Research Center, Department of Electrical and Computer Engineering, \\ the University of Texas at Austin, Austin TX, 78712-1084 U.S.A.
}

\begin{abstract}
Quantum dot photonic crystal lasers are demonstrated at room temperature by optical pulse pumping. Coupled cavities were designed based on square lattice PC slabs. Optimized two-dimensional photonic crystal cavities were defined in 200nm slabs with five-stacked InAs QDs layers. The two- and four-coupled cavities showed as low incident pump power threshold as $120 \mu \mathrm{W}$ and $370 \mu \mathrm{W}$, respectively, both from QD ground state emission range. Both clear threshold in pump power-output resonance power and resonance line width narrowing were observed from our membrane samples. The measured wavelengths matched very well with wavelengths predicted by 3D-Finite Difference Time Domain modelling.
\end{abstract}

Keywords: Quantum dot, Photonic crystal, Laser, Coupled cavity, Low threshold, Membrane

\section{INTRODUCTION}

Photonic crystal (PC) ${ }^{1,2}$ planar cavities can be compact building blocks, which will be used in future integrated nanophotonic systems. One of the most notable characters of PC cavities is that the mode profile and the quality $(Q)$ factors are geometrically controlled and can be designed. Simple defect cavity designs allowed the first photonic crystal lasers ${ }^{3}$ on InGaAsP multi-quantum well (QW) structures.

Quantum dot (QD)-PC lasers have some advantages as efficient light sources over QW-PC lasers. At first, QDs generally have smaller surface recombination rate than QWs. ${ }^{4}$ Negligible surface recombination is essential to thresholdless lasers. Secondly, the sharp homogeneous broadening emission of each QD can be fit in high- $Q$ PC resonances. ${ }^{5,6}$ At last, significant absorption loss by unnecessary active material can be minimized by putting single or several QDs only in a center of PC cavity. QD-PC lasers, however, have been more difficult devices to construct due to much lower available gain from QDs material. The smaller gain results not only from a lower total volume of actively emitting material but also from variations in spatial locality of QDs as well as inhomogeneous emission broadening consisting of narrow homogeneous broadening. These effects result in a smaller number of QDs contributing to the gain in a resonance as the $Q$ factor is increased.

We approach these issues by using high $Q$ cavities with relatively larger mode volumes. In this report, we describe simple designs of coupled cavities in square lattice(SQ) photonic crystals and demonstrate laser action from such quantum dot photonic crystal cavities.

\section{COUPLED PHOTONIC CRYSTAL CAVITIES}

Single defect square lattice slab cavity is found to have high- $Q$ whispering gallery $\operatorname{modes}^{6}(\mathrm{WGM})$. We started designing of QD-PC nano-lasers from the whispering gallery modes. The predicted mode volumes are generally larger due to the smaller band-gap. In order to increase optical gain $(\gamma)$ of QDs contributing to one mode, we employed coupled cavities ${ }^{7,8}$ based on the whispering gallery modes. A three-dimensional finite difference time domain (3D-FDTD) modelling was applied to structures shown in figure 1. Figure 1-(a), (b), and (c) show 2D SQ-PC slabs with single-, two- and four-defects, respectively. The analysis used 15 as lattice constant $(a)$ and

Further author information: (Send correspondence to T. Yoshie)

T.Yoshie: E-mail: yoshie@caltech.edu, Telephone: 16263952207 

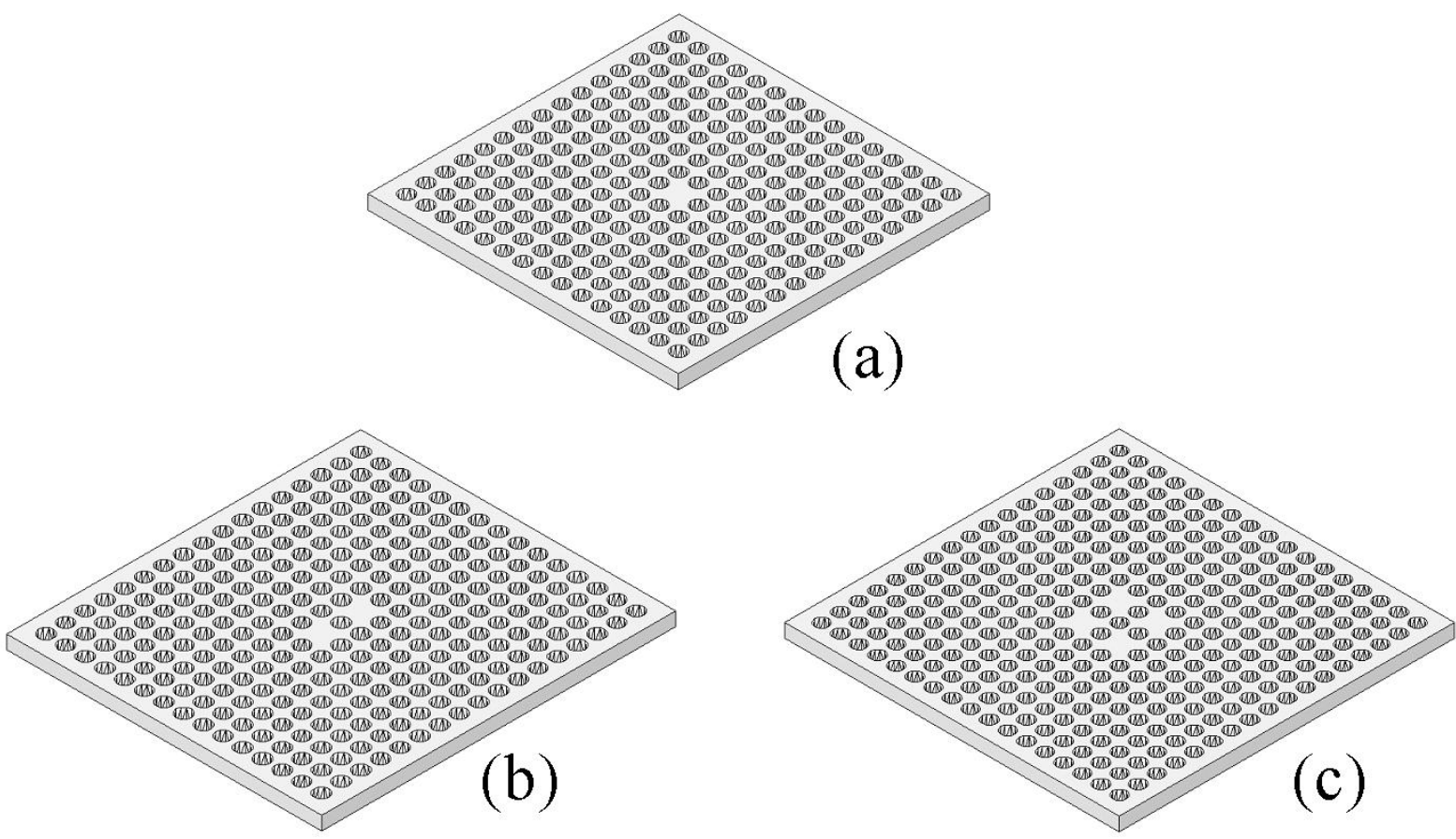

Figure 1.

PC Cavity structures used in this report. (a)single defect SQ cavity, (b)two-defects coupled cavity, and (c)fourdefects coupled cavity. The slab thickness is $d$. The lattice spacing is $a$.

3.4 as slab refractive index $(n)$. Identical defects overlapped in optical field lead to generation of supermodes, which cause splitting of eigenmode frequencies and symmetrically combined mode profiles. New eigenmodes $(\phi(r))$ of connected cavities can be described in a superposition form of each unperturbed cavity mode $\phi_{m}(r)$ with translation $r_{m}: \phi(r)=\Sigma_{m} A_{m} \phi_{m}\left(r-r_{m}\right)$. Our interests are even modes of electric field about a middle slice of slab(TM polarization). A good index to describe supermodes is z component of magnetic field since the magnetic field is always perpendicular to the slab on a middle of the slab for even modes. We chose even modes to obtain large dipole moment $\left(\mu_{E}\right)$ from InAs QDs.

Figure 2 shows three fourier spectra obtained from 3D-FDTD modelling on single-, two-, and four-defects coupled SQ-PC slab cavities. Dashed, solid and dotted lines represent single-, two- and four-defects coupled cavities. It can be noticed that a WGM resonance of single defect cavity is split symmetrically into two resonances about the WGM frequency on a spectrum of two-defects coupled cavity. In addition, the two supermode resonances from two-defects coupled cavity are split symmetrically into four supermodes indicated as arrows on four-defects coupled cavity spectrum.

Figure 3 shows amplitude profiles of x- and y-components of electric field $\left(E_{x}\right.$ and $\left.E_{y}\right)$ and z-component of magnetic field $\left(B_{z}\right)$ in a middle slice of the slab of single-defect SQ photonic crystal. The mode volume is generally ranged from 0.5 to $1 \times(\lambda / n)^{3}$, depending on the geometric parameters. Two defect coupled cavities were analyzed to map field profiles. The analyzed two-defects coupled cavities were located two lattice constants (a) apart from each other to form one coupled cavity in a 2D-square lattice PC slab with 15 by 17 lattice periods surrounding the cavities as shown in figure 1(b). The modelled photonic crystal slab geometry had a thickness $(d)$ of $0.73 a$, hole radii (r) of $0.36 a$. Figure 4 shows the $B z$ amplitude profiles in the middle slice of the slab. You can notice symmetric features of even and odd supermodes in the figures. The electric field is vertical to the hole cylinder axis in the middle of the slab. The calculated vertical and the lateral $Q$ factors $\left(Q_{\perp}\right.$ and $\left.Q_{\|}\right)$, the mode volume $\left(V_{\text {mode }}=\int\left(\epsilon(r) \mathrm{E}(\mathrm{r})^{2}\right) \mathrm{dr}^{3} / \max \left(\epsilon\left(r^{\prime}\right) \mathrm{E}\left(\mathrm{r}^{\prime}\right)^{2}\right)\right)$ are shown in table 1 . 
Table 1. Performance of a whispering gallery mode from a single defect cavity and two supermodes from two-defects coupled cavity. 3D-FDTD simulation was used for the analysis. The unit of mode volume $\left(V_{\text {mode }}\right)$ is in $(\lambda / n)^{3}$.

\begin{tabular}{|l|l|l|l|l|l|}
\hline SQ-PC cavity & mode type & $Q_{\|}$ & $Q_{\perp}$ & $V_{\text {mode }}$ & $a / \lambda$ \\
\hline Two defects & Even supermode of WGMs & 57000 & 28000 & 1 & 0.2892 \\
& Odd supermode of WGMs & 39000 & 24000 & 1 & 0.2861 \\
Single defect & WGM & 77000 & 29000 & 0.7 & 0.2874 \\
\hline
\end{tabular}

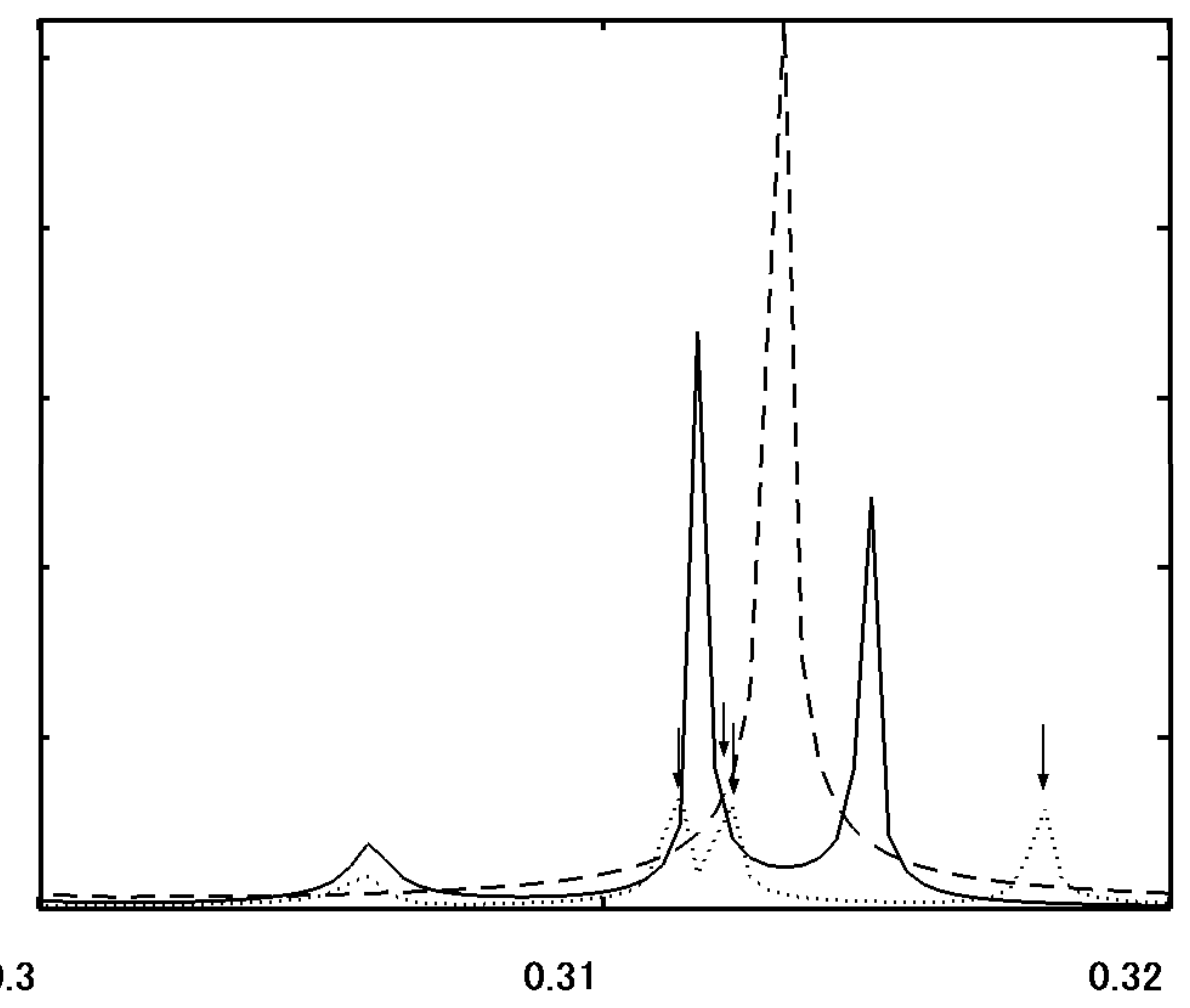

Figure 2.

Fourier spectra obtained from 3D-FDTD modelling on single-, two-, and four-defects SQ-PC slab cavities. Dashed, solid and dotted lines are analyzed from single, two, and four defects SQ-PC slab cavities. The analyzed parameters are $r=0.38 a, d=0.47$ and $n=3.4$. Arrows indicated on the fourier spectrum are four supermode resonances of four defects coupled cavity.

As shown in the table 1, two-defects coupled cavities therefore increase the mode volume by $50 \%$ and we can generate high- $Q$ supermodes by coupled cavities with slight decrease in the $Q$ factor. By putting the defects more away from each other, the high- $Q$ factors of single defect mode can be preserved on coupled cavities with much smaller interaction of modes and the mode volume approaches two times as big as single defect cavity WGM. The frequency separation of supermodes would be decreased as the interacted fields decrease. Supermodes can be used for optical switches and lasers with more distributed mode profiles. 

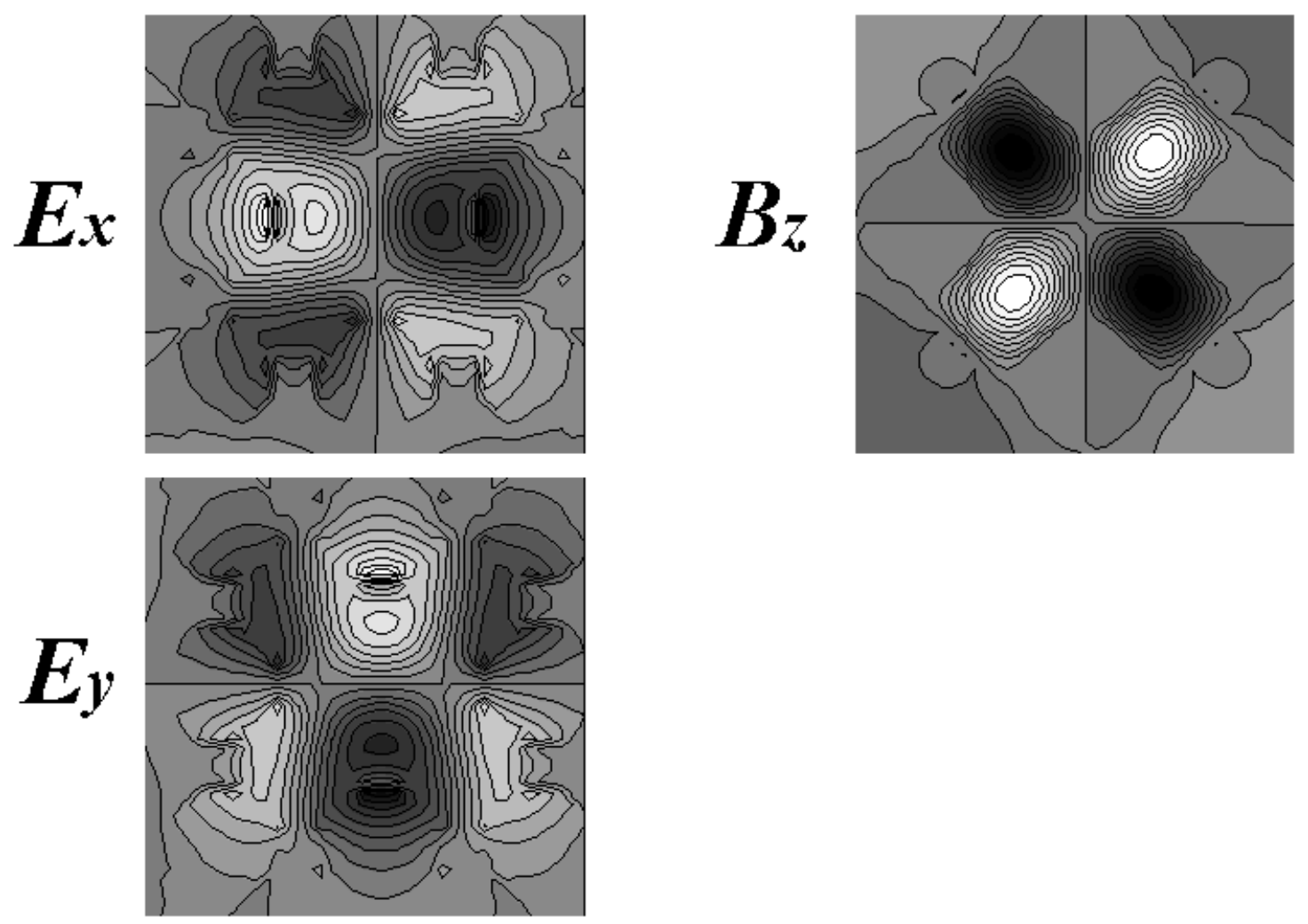

Figure 3.

Amplitude profiles of $\mathrm{x}$ and $\mathrm{y}$ components of electric field ( $E_{x}$ and $\left.E_{y}\right)$ and $\mathrm{z}$ component of magnetic field $\left(B_{z}\right)$ analyzed from single-defect SQ-PC cavity. Black and white regions have intense amplitudes, but have different signs of amplitudes. The analyzed lattice parameters are $r=0.36 a, d=0.73 a$ and $n=3.4$. On the other hand, $E_{z}, B_{x}$ and $B_{y}$ components are negligible on the simulation results, which are predicted by symmetric analysis for even modes. 


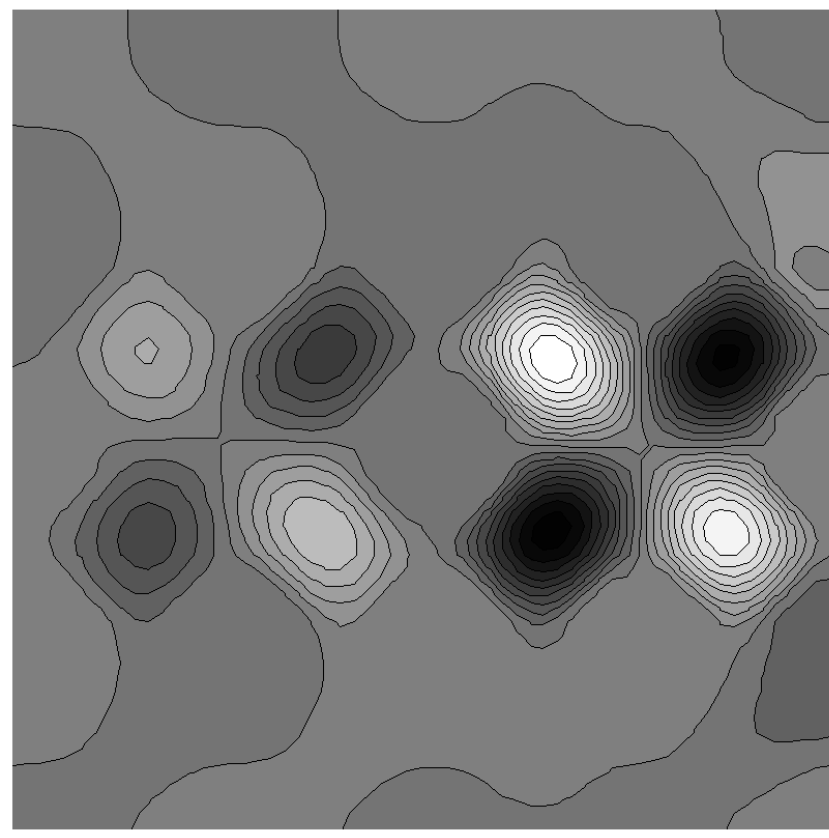

(a)

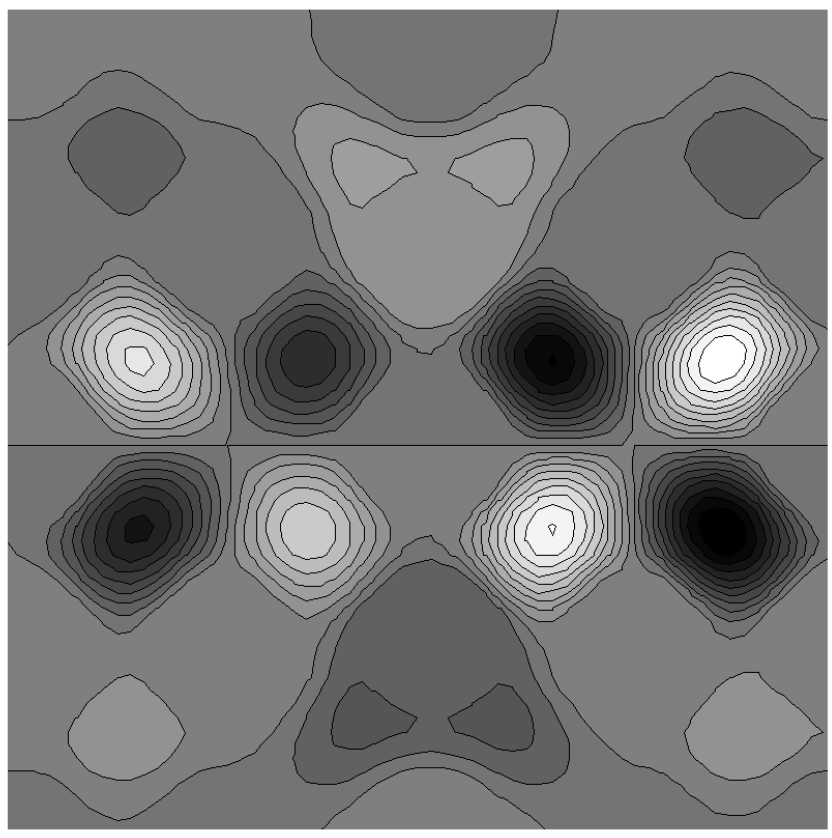

(b)

Figure 4.

Z-component of magnetic field (Bz) on a middle slice of two-defects coupled cavity slab. (a) and (b) show $B z$ mappings from odd (left) and even (right) supermodes. Black and white regions have intense amplitudes, but have different signs of amplitudes. The analyzed lattice parameters are $r=0.36 a, d=0.73 a$ and $n=3.4$. Similarly with results of single-defect SQ-PC cavity, $E_{z}, B_{x}$ and $B_{y}$ components are negligible on the simulation results.

\section{QUANTUM DOT PHOTONIC CRYSTAL LASERS}

\subsection{Sample preparation}

Five stacked self-assembled InAs QDs layers were grown by molecular beam epitaxy. The QD density in our samples is $5 \times 10^{10} / \mathrm{cm}^{2}$ in total and the QD layers were separated by $30 \mathrm{~nm}$ GaAs layers. $\mathrm{Al}_{0.2} \mathrm{Ga}_{0.8} \mathrm{As}_{\text {is }}$ used for cladding layers to form thin slab waveguides, which are deposited on an $800 \mathrm{~nm} \mathrm{Al}_{0.94} \mathrm{Ga}_{0.06} \mathrm{As}$ sacrificial layer on a top of GaAs substrate. In order to define 200nm-thick 2D-PC slab cavities, electron-beam lithography, chemically assisted ion-beam etching, oxidation of $\mathrm{Al}_{0.94} \mathrm{Ga}_{0.06}$ As layer, and wet etching of $\mathrm{AlOx}$ layer were performed. The detailed fabrication steps can be found elsewhere. ${ }^{9}$ Many geometries of single-, two-, and four-defects coupled cavities were fabricated within photonic crystals with lattice sizes of 31 by 31,31 by 33 , and 33 by 33 , respectively.

\section{2. $\mu$-photoluminescence}

Figure 5 shows measurement results of $\mu$-photoluminescence. Single defect cavity has one intense resonance from WGM around 1330nm. Two-defects coupled cavity has two intense supermode resonances and one weak resonance in lower frequency. Four-defects coupled cavity has four intense supermode resonances in addition to one weak resonance around $1370 \mathrm{~nm}$. The weak resonance is located at a dielectric band edge. These spectra match with simulation results very well. 


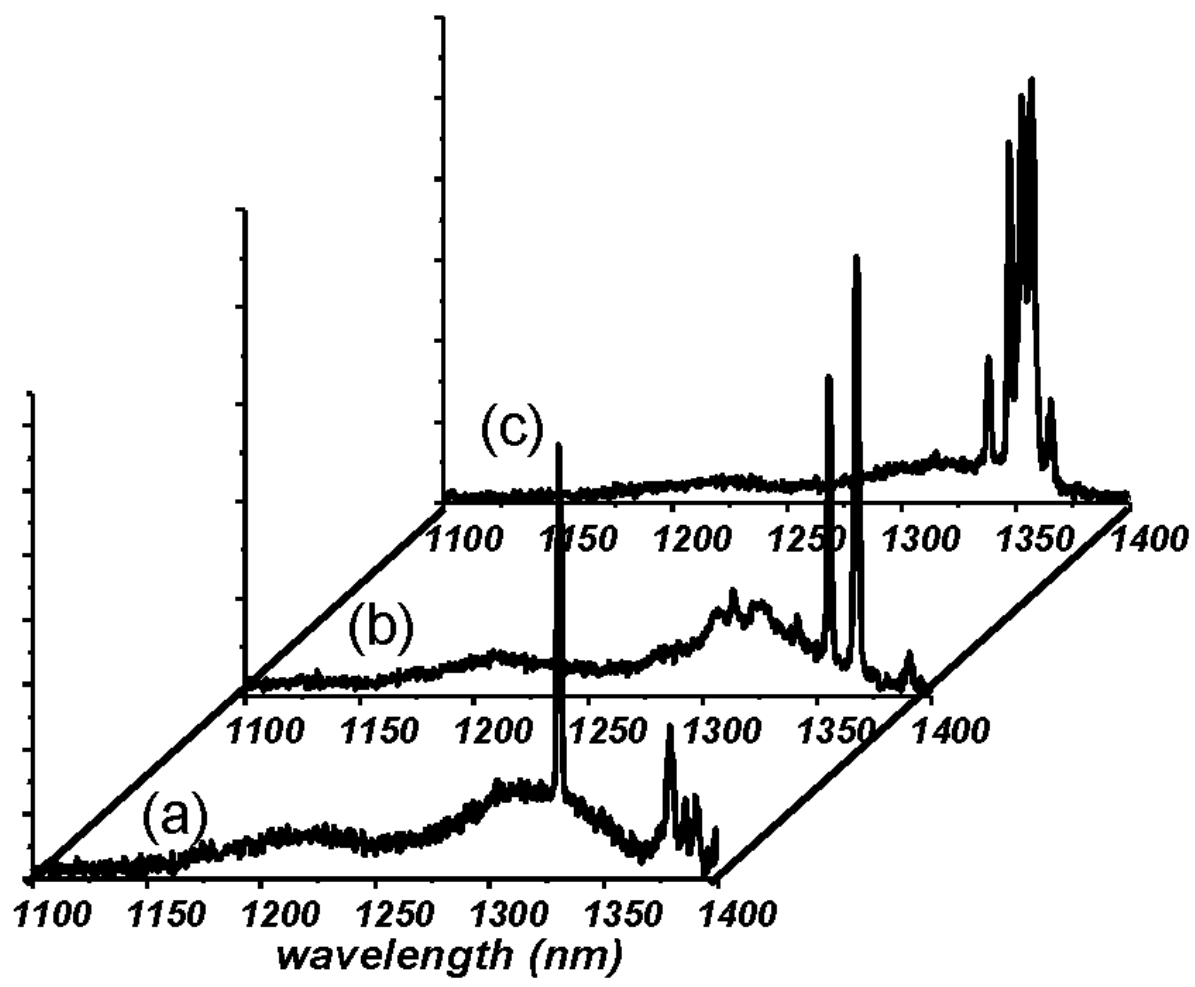

Figure 5.

$\mu$-photoluminescence spectra taken from single-, two- and four-defects SQ PC slab cavities, respectively, from a bottom of the figure.

\subsection{Pulsed-optical pumping of SQ-PC cavities}

A pulsed 780nm semiconductor laser diodes was used to measure luminescence spectra and $L_{\text {pump }}$ (pump power)$L_{\text {out }}$ (collected resonance power) curves. Twenty nanosecond pulses with $2.86 \%$ duty cycle were applied to the pumping laser diodes. The pump light was confirmed to have good pulse shapes in time domain. The pumping beam diameter was varied up to $4 \mu \mathrm{m}$. Figure 6-(1) shows two L-L curves taken from two- and four-defects coupled cavities. In an inset of the figure 6 you can find scanning electron microscope images of two-defects and four-defects coupled cavities. Each of these cavities exhibits distinct threshold and linear increase in output power above threshold. Threshold pump powers of $120 \mu \mathrm{W}$ and $370 \mu \mathrm{W}$ are measured for two- and four-defects coupled cavities, respectively. Considering filling factors and transmission of pump light at air/semiconductor interfaces, the pump light power in the membranes would be approximately only $15 \%$ of incident pump power with perfect absorption by cavities. Fifteen percentages of threshold incident pump powers are $18 \mu \mathrm{W}$ and $56 \mu \mathrm{W}$ for two defects and four defects coupled cavities, respectively. Actual absorbed pump power would be smaller than this estimation due to imperfect absorption. These facts allow us to believe that our nanolasers are operated by very small power and that sub- $\mu \mathrm{W}$ threshold lasers could be constructed by improvement of fabrication. Figure 6-(2) shows luminescence spectra from a four-defects coupled cavity. Below threshold (Fig.6 (2-a)), the resonance cannot be clearly observed. Close to threshold, nonlinear amplification of emission is seen (Fig.6 (2-b)). Above threshold (Fig.6 (2-c)), the resonance intensity increases significantly. The lasing wavelength of $1328 \mathrm{~nm}$ matches the QDs ground state emission. The lasing frequency of $a / \lambda=0.316$ in this laser also matches the analyzed mode frequency of two-defects coupled cavities. Spectral line widths decreased from 1-2 $\mathrm{nm}$ (below threshold) to less than $0.2 \mathrm{~nm}$ (above threshold). With moderate $\mathrm{CW}$ pumping, the resonance showed $\lambda / \Delta \lambda=2100$. The combination of distinct threshold in the $L_{\text {pump }}-L_{\text {out }}$ curves and line width narrowing both indicate that the cavities are definitely lasing. ${ }^{10}$ For two-defects coupled cavities, the 
(1)

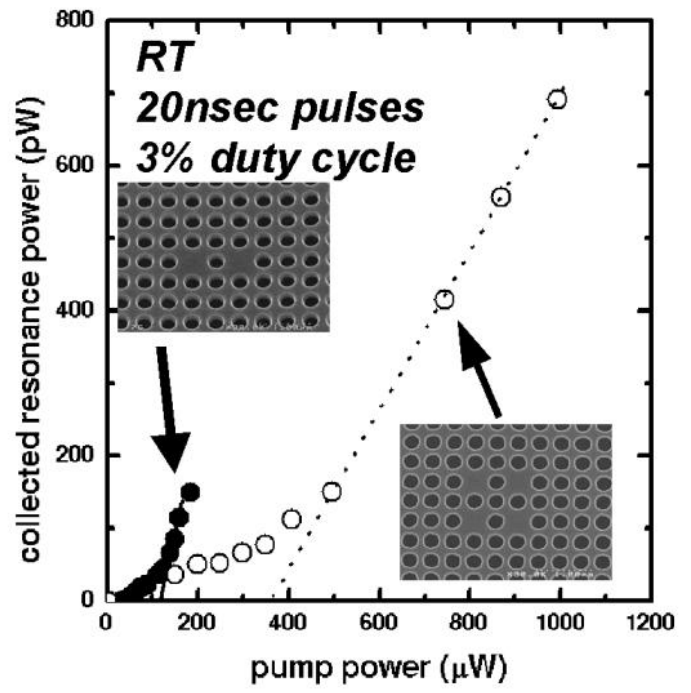

(2)

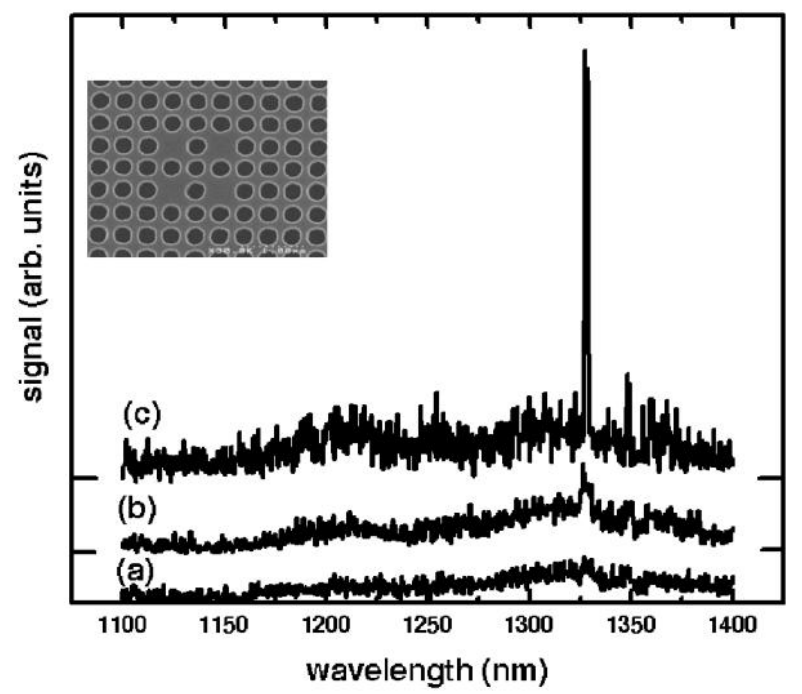

Figure 6.

(1) L-L characteristics of two-defects and four-defects coupled cavities, (2) Luminescence spectra taken from four-defects coupled cavity. These measurements were performed by optical pulse pumping (20nsec, $2.86 \%$ duty cycle) with $780 \mathrm{~nm}$ semiconductor laser diodes at room temperature $\left(23^{\circ} \mathrm{C}\right)$.

resonance was measured at $a / \lambda=0.3$. The simulated mode volumes are $0.8(\lambda / n)^{3}$ and $1 \times(\lambda / n)^{3}$, respectively, by using geometric parameters of lased PC samples. Single defect cavities did not lase in our experiments, probably due to a lack of sufficient gain from the QDs, but the single defect cavities have strong possibilities of lasing from our simulation results.

\section{ACKNOWLEDGMENTS}

The authors gratefully appreciate valuable discussions from Marko Loncar. This work was supported by the NSF under Grant ECS-9912039 and DMR-0103134, the AFOSR under F49620-01-1-0497, and by DARPA under MDA972-00-1-0019.

\section{REFERENCES}

1. E. Yablonovitch, "Inhibited spontaneous emission in solid-state physics and electronics," Phys. Rev. Lett. 58, pp. 2059-2062, 1987.

2. S. John, "Strong localization of photons in certain disordered dielectric superlattices," Phys. Rev. Lett. 58, pp. 2486-2489, 1987.

3. O. Painter, R. Lee, A. Scherer, A. Yariv, J. O'Brien, P. Dapkus, and I. Kim, "Two-dimensional photonic band-gap defect mode laser," Science 284, pp. 1819-1821, 1999.

4. M. Boroditsky, I. Gontijo, M. Jackson, R. Vrijen, E. Yablonovitch, T. Krauss, C. Cheng, A. Scherer, R. Bhat, and M. Krames, "Surface recombination measurements on iii-v candidate materials for nanostructure light-emitting diodes," J. Appl. Phys. 87, pp. 3497-3504, 2000.

5. J. Vuckovic, M. Loncar, H. Mabuchi, and A. Scherer, "Design of photonic crystal microcavities for cavity qed," Phys. Rev. E 65, pp. 016608(1)-(11), 2002. 
6. H. Ryu, S. Kim, H. Park, J. Hwang, Y. Lee, , and J. Kim, "Square-lattice photonic band-gap single-cell laser operating in the lowest-order whispering gallery mode," Appl. Phys. Lett. 80, pp. 3883-3885, 2002.

7. A. Yariv, Y. Xu, R. Lee, and A. Scherer, "Coupled-resonator optical waveguides," Opt. Lett. 24, pp. 711713, 1999.

8. M. Bayindir, B. Temelkuran, and E. Ozbay, "Propagation of photons by hopping: A waveguiding mechanism through localized coupled-cavities in three-dimensional photonic crystals," Phys. Rev. B 61, pp. R11855-R11858, 2000.

9. T. Yoshie, J. Vuckovic, A. Scherer, H. Chen, and D. Deppe, "High quality two-dimensional photonic crystal slab cavities," Appl. Phys. Lett. 79, pp. 4289-4291, 2001.

10. T. Yoshie, O. Schchekin, H. Chen, D. Deppe, and A. Scherer, "Quantum dot photonic crystal lasers," Electron. Lett. 38, pp. 967-968, 2002. 